\title{
Wearable laser Doppler sensors for evaluating the nutritive and shunt blood flow
}

Loktionova, Yulia, Zharkikh, Elena, Zherebtsov, Evgeny, Kozlov, Igor, Sidorov, Viktor, et al.

Yulia I. Loktionova, Elena V. Zharkikh, Evgeny A. Zherebtsov, Igor O. Kozlov, Viktor V. Sidorov, Angelina I. Zherebtsova, Sergei G. Sokolovski, Andrey V. Dunaev, Edik U. Rafailov, "Wearable laser Doppler sensors for evaluating the nutritive and shunt blood flow," Proc. SPIE 11457, Saratov Fall Meeting 2019: Optical and Nano-Technologies for Biology and Medicine, 114570M (9 April 2020); doi: 10.1117/12.2564396

EDent: Saratov Fall Meeting 2019: VII International Symposium on Optics and Biophotonics, 2019, Saratov, Russian Federation 


\title{
Wearable laser Doppler sensors for evaluating the nutritive and shunt blood flow
}

\author{
Yulia I. Loktionova ${ }^{a}$, Elena V. Zharkikh ${ }^{\mathrm{a}}$, Evgeny A. Zherebtsov ${ }^{\mathrm{b}}$, Igor O. Kozlov ${ }^{\mathrm{a}}$, \\ Viktor V. Sidorov ${ }^{c}$, Angelina I. Zherebtsova ${ }^{a}$, Sergei G. Sokolovski ${ }^{\mathrm{d}}$, \\ Andrey V. Dunaev ${ }^{\mathrm{a}}$, and Edik U. Rafailov ${ }^{\mathrm{d}, \mathrm{e}}$ \\ ${ }^{a}$ Research and Development Center of Biomedical Photonics, Orel State University named \\ after I.S. Turgenev, Orel, Russia \\ ${ }^{\mathrm{b}}$ Optoelectronics and Measurement Techniques, University of Oulu, Oulu, FI-90014, Finland \\ "SPE "LAZMA" Ltd, Moscow, Russia \\ dAston Institute of Photonic Technologies, Aston University, Birmingham, UK \\ eInternational Center of Critical Technologies in Medicine, Saratov State University, Saratov, \\ Russia
}

\begin{abstract}
This study is devoted to the trials of wearable diagnostic system that implements the laser Doppler flowmetry technique to analyse the blood microcirculation. We do preliminary test with involvement of limited group of healthy volunteers of different age and in patients with type 2 diabetes. During the series of measurements, the microcirculation parameters was measured for 10 minutes in the palmar surfaces of the big toes and in the inner sides of the upper thirds of the shins. A statistically significant differences was found in bypass index, nutritive and shunt blood flow in shins between older group of volunteers and patients's group as well as in shunt blood flow in fingers between younger and older groups of volunteers.
\end{abstract}

Keywords: laser Doppler flowmetry, microcirculation, ageing, diabetes mellitus, nutritive and shunt blood flow, bypass index

\section{INTRODUCTION}

The number of people with diabetes mellitus (DM) is increasing every year. According to estimates by the International Diabetes Federation, in 2017 the number of such people amounted to 425 million, and a further 1.5 times increase is predicted by 2045. ${ }^{1}$ Diabetes mellitus can cause a large number of complications in the patient's body, which can lead to a decrease in the quality of life of patients and, as a result, even to death. Some of the most common complications are limb problems, including diabetic foot syndrome, neuropathy, loss of sensitivity. That complications are caused by disorders in the cardiovascular system, starting with the microcirculatory bed and progressing at an alarming rate every year in the absence of proper treatment. Currently, there are several painless, safe, non-invasive optical methods for assessing the state of microcirculation e.g. based on skin temperature measurements ${ }^{2,3}$ or hyperspectral imaging. ${ }^{4}$ Quite widespread methods for non-invasive blood flow measurements are ones based on the principles of dynamic light scattering (DLS). The family of methods can be used to do measurements both in vitro ${ }^{5}$ and in vivo. ${ }^{6,7}$ In this work we employ the laser Doppler flowmetry (LDF) technique. The approach uses the principles of DLS and is based on the laser sensing of tissue and the analysis of the reflected light scattered in static tissue and in moving red blood cells. The sampling volume of the measurements changes during pulse beat and can achieve $1-2 \mathrm{~mm}^{3}{ }^{8}$. The outcome result of the measurement technique is the LDF-gram recording (an example of one is presented in figure 1, A), which shows the development of the blood flow changes in time..$^{9,10}$

The method also allows ones to assess the state of local regulatory mechanisms since the recorded signal is an overlay of several oscillatory processes. Estimation of the contribution of different oscillations to the overall

Send correspondence to Yulia Loktionova

E-mail: julya-loktionova@mail.ru 

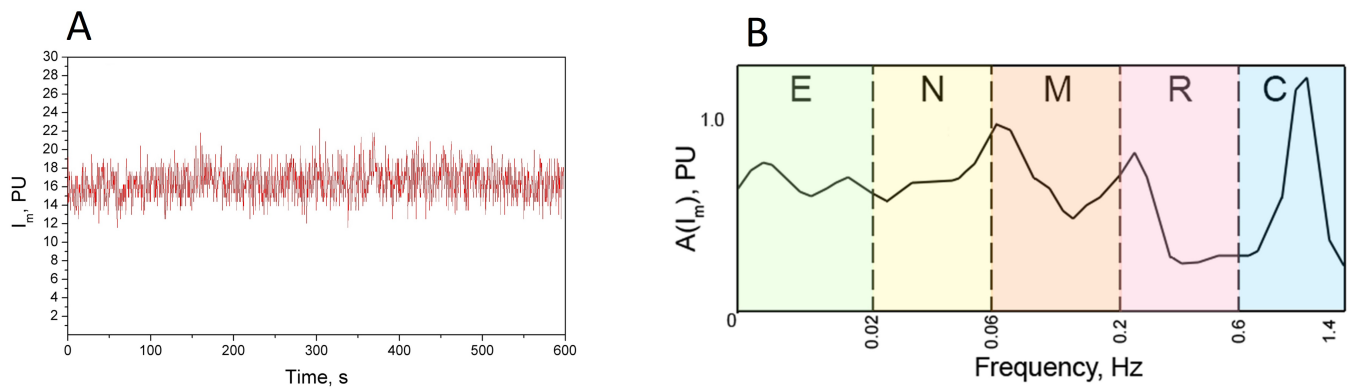

Figure 1: The typical example of LDF-record (A) and a wavelet analysis of the obtained signal (B)

signal level is possible by means of LDF signal processing by wavelet analysis. Currently, there are five main factors, which have its impact in a certain frequency range. Endothelial oscillations $(0.0095-0.021 \mathrm{~Hz})$ are due to the activity of the cells of the inner layer of blood vessels, neurogenic $(0.021-0.052 \mathrm{~Hz})$ - are formed under the influence of the nervous regulation of blood vessels, myogenic oscillations $(0.052-0.145 \mathrm{~Hz})$ are the result of the activity of vascular smooth muscle, respiratory $(0.145$ to $0.6 \mathrm{~Hz})$ and cardiac $(0.6$ to $2 \mathrm{~Hz})$ oscillations are generated through the distribution of the vessels of the respiratory and pulse waves ${ }^{11}$ (Fig.1 B).

With the help of the LDF method, it is possible to assess not only the presence of violations or their absence, but also the degree of damage by changes in the microcirculation. ${ }^{12-14}$ It was found that the violations in the microvasculature may occur before clinical manifestations of diabetes. ${ }^{15}$ The complexity of determining the exact state of human microcirculation in pathologies is that the microvasculature is not stable during human life. ${ }^{16}$ The microcirculation bed changes under the influence of external factors, including age. So, that is crucial to determine the limits of reference values of parameters for different age groups.

The purpose of this work was to investigate the microcirculation system and to assess the nutritional and shunt flow in lower limbs in patients with diabetes in comparison with healthy volunteers.

\section{MATERIALS AND METHODS}

Experimental studies were carried out using four wearable laser Doppler flowmetry monitors "AMT-LAZMA 1" (Aston Medical Technology Ltd., UK) for blood microcirculation analysis. The single-mode VCSEL laser operating at a wavelength of $850 \mathrm{~nm}$ is used as an emitter in the devices. The devices also have a built-in temperature sensor and accelerometer.

The inner parts of the upper thirds of the shins and the palmar surface of big toes, were chosen as places of study (Fig.2). These points were chosen based on the fact that the skin in these places belongs to different types: in the area of the big toes - skin with a large number of arteriole-venular anastomoses (AVA), and in the area of the shins - skin without AVA. ${ }^{17}$

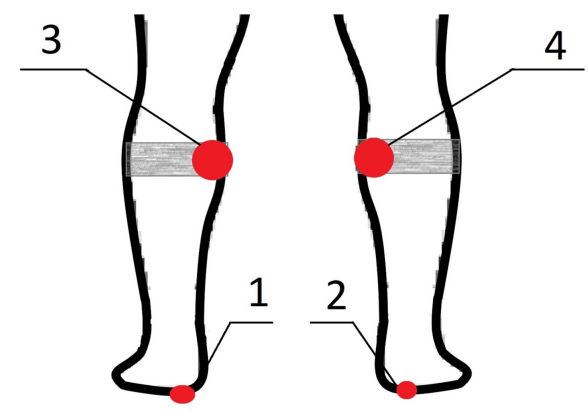

Figure 2: Localization of sensors (1 - right big toe; 2 - left big toe; 3 - right shin; 4 - left shin) 
The index of microcirculation $(\mathrm{Im})$ was recorded for 10 minutes, with the volunteers and patients lying on a couch. Studies were conducted in a state of complete physical and psychological rest, not earlier than 2 hours after eating and medical procedures. Also, the research was conducted in accordance with the principles set out in the Helsinki Declaration of 2013 by the World Medical Association and was approved by the Ethics Committee of Orel State University.

The experiments involved 2 groups of healthy volunteers without cardiovascular and other diseases and the third group of participants included patients with type 2 diabetes mellitus. Healthy volunteers were divided into two age groups so that patients were the same age as one of the volunteers groups, and another group of volunteers was significantly younger. The number of study participants and their average age are presented in Table 1. There was no statistically significant difference in blood pressure and heart rate between the groups of healthy volunteers.

Table 1: Groups of volunteers and patients

\begin{tabular}{|c|l|l|l|}
\hline Localization of sensors & 1 group (younger) & 2 group (older) & Patients \\
\hline Shins & - & $\begin{array}{l}9 \text { volunteers } \\
(52.3 \pm 10.7 \text { years })\end{array}$ & $\begin{array}{c}16 \text { patients } \\
(57.2 \pm 9.1 \text { years })\end{array}$ \\
\hline Big toes & 17 volunteers & 10 volunteers & 43 patients \\
& $(21.7 \pm 1.4$ years $)$ & $(51.8 \pm 14.4$ years $)$ & $(56.6 \pm 11.8$ years $)$ \\
\hline
\end{tabular}

To assess the state of the microcirculatory system and obtain quantified diagnostic information, the bypass index $(B I)$ was evaluated, as well as nutritional and shunt blood flow parameters according to the procedure described by Dunaev et al. ${ }^{18}$ The total value of the $B I$ is obtained by adding $B I_{1}$ and $B I_{2}$, where $B I_{1}$ is the shunting index associated with differences in tone in the microvessels of the nutritive and non nutritive blood flow pathways directly within the microcirculatory bed, and $B I_{2}$ is the shunting index associated with differences in perfusion of microvessels and larger vascular segments (arteries, venules and veins) in cases of arterial hyperemia or venous stagnation: ${ }^{19}$

$$
B I_{1}=A n / A m
$$

where $A n$ and $A m$ - respectively maximum amplitudes of oscillations of neurogenic and myogenic frequency ranges (for zones with AVA).

$$
B I_{1}=A \max / A m
$$

where $A$ max - the maximum amplitude of active oscillations (for zones without AVA). $B I_{2}$ is calculated in the same way for zones with AVA and without AVA.

$$
B I_{2}=A c(r) / A m,
$$

where $A c(r)$ - the dominant amplitude of oscillations of the heart and respiratory rhythms. It is taken into account if it is greater than or equal to 1 . By the calculation of $B I$, it is possible to assess the perfusion of nutritive and shunt pathways in microvascular networks. In areas with AVA the value of nutritive perfusion (Mnutr) is calculated by the expression:

$$
\text { Mnutr }=\operatorname{Im} /(1+B I)
$$

For areas without AVA:

$$
\text { Mnutr }=I m / B I .
$$

Accordingly, the value of shunt perfusion (Mshunt) is estimated by the expression:

$$
\text { Mshunt. }=\text { Im }- \text { Mnutr. }
$$




\section{RESULTS AND DISCUSSION}

Between older volunteers and patients with type 2 diabetes a statistically significant difference was found in the bypass index, nutritive and shunt blood flow (Fig.3, A, B). The rate of bypass index in patients is higher than in older volunteers, while nutritional and shunt blood flow is lower. In the big toes, a statistically significant difference in shunt blood flow was also found between younger volunteers and patients (Fig.3 C).

A

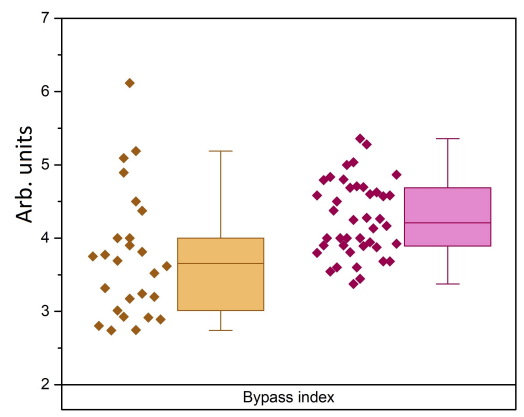

B

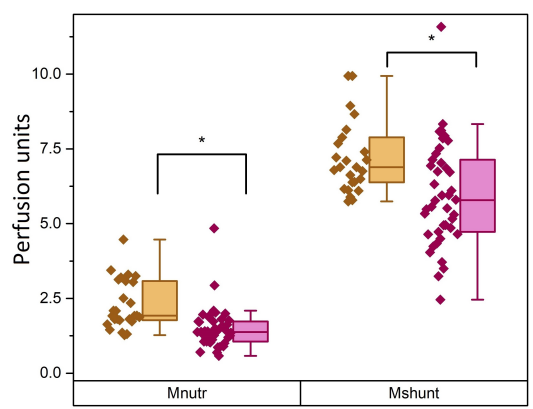

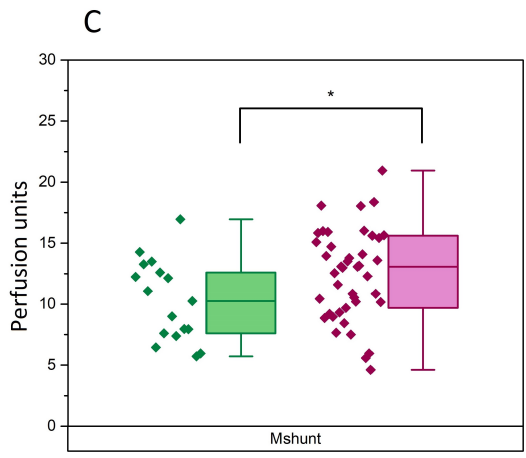

Figure 3: Results of analysis in shins (A, B) and in fingers (C) of younger volunteers (green boxes), older volunteers (yellow boxes) and patients (purple boxes) A - calculation of bypass index, B - calculation of nutritive and shunt blood flow, $\mathrm{C}$ - calculation of shunt blood flow.

* - The significance of the difference between the values was confirmed with $p<0.05$ according to the MannWhitney test

These results may suggest that age and diabetes affect the microcirculation in the same way with the slower development of age-related changes and their differences can be identified when compared with younger volunteers.

\section{CONCLUSIONS}

Thus, the calculation of additional parameters of microcirculation allows ones to create a more complete picture of the cardiovascular state in both a healthy person and a patient with diabetes. By calculating the bypass index, nutritive and shunt blood flow, it is possible to estimate the index of microcirculation in the nutritive and shunt pathways. It also allows ones to set the boundaries of reference values in healthy volunteers of different age groups in different places of measurement.

\section{ACKNOWLEDGMENTS}

Clinical studies and data processing (EVZ, YL, IK, AZ) were funded by the Russian Science Foundation (the research project 18-79-00237). ER and SS acknowledges support from the research project 18-15-00172.

\section{REFERENCES}

[1] "Idf diabetes atlas," Tech. Rep. 8 (2017).

[2] Zherebtsov, E. A., Zherebtsova, A. I., Doronin, A., Dunaev, A., Podmasteryev, K. V., Bykov, A., and Meglinski, I., "Combined use of laser Doppler flowmetry and skin thermometry for functional diagnostics of intradermal finger vessels," Journal of Biomedical Optics 22(4), 1 - 4 (2017).

[3] Sagaidachnyi, A., Fomin, A., Usanov, D., and Skripal, A., "Real-time technique for conversion of skin temperature into skin blood flow: human skin as a low-pass filter for thermal waves," Computer Methods in Biomechanics and Biomedical Engineering 22, 1-11 (05 2019). 
[4] Zherebtsov, E., Dremin, V., Popov, A., Doronin, A. Kurakina, D., Kirillin, M., Meglinski, I., and Bykov, A., "Hyperspectral imaging of human skin aided by artificial neural networks," Biomedical Optics Express 10, 3545-3559 (07 2019).

[5] Nepomnyashchaya, E., Aksenov, E., Bogomaz, T., and Velichko, E., "Use of laser correlation spectroscopy to investigate the parameters of biological suspensions," Journal of Optical Technology 82, 162 (03 2015).

[6] Dremin, V., Kozlov, I., Volkov, M., Margaryants, N., Potemkin, A., Zherebtsov, E., Dunaev, A., and Gurov, I., "Dynamic evaluation of blood flow microcirculation by combined use of the laser doppler flowmetry and high-speed videocapillaroscopy methods," Journal of Biophotonics 12 (01 2019).

[7] Mizeva, I., Zharkikh, E., Dremin, V., Zherebtsov, E., Makovik, I., Potapova, E., and Dunaev, A., "Spectral analysis of the blood flow in the foot microvascular bed during thermal testing in patients with diabetes mellitus," Microvascular Research 120, 13-20 (05 2018).

[8] Dremin, V., Zherebtsov, E., Bykov, A., Popov, A., Doronin, A., and Meglinski, I., "Influence of blood pulsation on diagnostic volume in pulse oximetry and photoplethysmography measurements," Applied Optics $\mathbf{5 8}$ (2019).

[9] Mizeva, I., Dremin, V., Potapova, E., Zherebtsov, E., Kozlov, I., and Dunaev, A., "Wavelet analysis of the temporal dynamics of the laser speckle contrast in human skin," IEEE Transactions on Biomedical Engineering , 1-1 (2019).

[10] Fredriksson, I., Larsson, M., and Strömberg, T., "Model-based quantitative laser Doppler flowmetry in skin," Journal of Biomedical Optics 15(5), 1 - 12 (2010).

[11] Lancaster, G., Stefanovska, A., Pesce, M., Marco Vezzoni, G., Loggini, B., Pingitore, R., Ghiara, F., Barachini, P., Cervadoro, G., Romanelli, M., and Rossi, M., "Dynamic markers based on blood perfusion fluctuations for selecting skin melanocytic lesions for biopsy," Scientific Reports 5, 12825 (2015).

[12] Mahgoub, M. A. and Abd-Elfattah, A. S., "Diabetes mellitus and cardiac function," Molecular and Cellular Biochemistry 180, 59-64 (Mar 1998).

[13] Donnelly, R., Emslie-Smith, A. M., Gardner, I. D., and Morris, A. D., "Vascular complications of diabetes," $B M J$ 320(7241), 1062-1066 (2000).

[14] Aastha Chawla, R. C. and Jaggi, S., "Microvasular and macrovascular complications in diabetes mellitus: Distinct or continuum?," Indian Journal of Endocrinology and Metabolism (2016).

[15] Hu, H.-F., Hsiu, H., Sung, C.-J., and Lee, C.-H., "Combining laser-doppler flowmetry measurements with spectral analysis to study different microcirculatory effects in human prediabetic and diabetic subjects," Lasers in Medical Science 32, 327-334 (Feb 2017).

[16] Makrantonaki, E. and Zouboulis, C., "Characteristics and pathomechanisms of endogenously aged skin," Dermatology 214(4), 352-360 (2007).

[17] Urbancic-Rovan, V., Stefanovska, A., Bernjak, A., Azman-Juvan, K., and Kocijancic, A., "Skin blood flow in the upper and lower extremities of diabetic patients with and without autonomic neuropathy," Journal of Vascular Research 41(6), 535-545 (2004).

[18] Dunaev, A. V., Sidorov, V. V., Krupatkin, A. I., Rafailov, I. E., Palmer, S. G., Stewart, N. A., Sokolovski, S. G., and Rafailov, E. U., "Investigating tissue respiration and skin microhaemocirculation under adaptive changes and the synchronization of blood flow and oxygen saturation rhythms," Physiological Measurement 35, 607-621 (mar 2014).

[19] Krupatkin, A. I., "Evaluation of the parameters of total, nutritive, and shunt blood flows in the skin microvasculature using laser doppler flowmetry," Fiziologiia cheloveka (2005). 